\title{
Planificación de Sistemas de Información en la Empresa: El Intercambio Electrónico de Datos (EDI)
}

\author{
Carlos Serrano, Luis Alberto Fabra y Emma Lobera \\ Universidad de Zaragoza \\ Departamento de Contabilidad y Finanzas
}

\subsection{Resumen}

El EDI consiste en transmitir electrónicamente documentos comerciales y administrativo-contables entre aplicaciones informáticas en un formato normalizado, de forma las empresas puedan procesar dichos documentos sin intervención manual. En este trabajo describimos los sistemas de información electrónicos, las diferentes normas EDI que existen, los beneficios y los costes que para una empresa puede suponer el implantar estos sistemas y el impacto que puede tener sobre la gestión empresarial. Hemos clasificado este impacto en factores relativos a la gestión de los documentos, factores logísticos, estratégicos y organizativos, factores relativos a la seguridad y finalmente, las implicaciones legales y fiscales del EDI. (Autor)

Palabras clave: Intercambio Electrónico de Datos. Sistemas de Información Contables.

\subsection{Abstract}

EDI is a standard for transmiting commercial, administrative and accounting documents among informatic applications, so that firms can proccess these documents without human intervention. This article, describes electronic information systems, the different EDI implementations, the different costs and profits that firms should expect, and its impact on management. This impact has been classified in four categories: a) factors related to document management; b) logistic factors, strategic and organizative factors; c) security questions; and finally, d)legal and fiscal implications.

Keywords: Electronic Data Interchange. Accounting information systems 


\section{Introducción}

Hoy en día, el sistema informativo contable de cualquier empresa, por pequeña que sea, se encuentra informatizado, de forma que habitualmente se logran unos elevados niveles de automatización en las tareas administrativas. Por ejemplo, es frecuente que se encuentren integrados los programas de contabilidad con los que gestionan la tesorería o el inventario y que estos datos se procesen muy rápidamente. Aunque es menos habitual, las empresas pueden disponer de complejos sistemas de información para la gestión e incluso de modernos sistemas de información para la dirección, perfectamente integrados con los anteriores.

Pero suele suceder que dos empresas que mantienen una intensa relación comercial cliente-proveedor y que disponen de los sistemas de información contables más avanzados, ejecuten sus transacciones empresariales introduciendo las órdenes de compra, las facturas, y el resto de documentos en sobres, que posteriormente son enviados por correo. También es habitual enviar estos documentos a través del fax, con lo que se agiliza la gestión. Si la empresa utiliza un faxmodem conectado al ordenador y gestionado por un programa informático, se evita tener que imprimir los documentos, enviándolos directamente desde su ordenador hasta el fax de la otra empresa, aunque, en cualquier caso hay que volver a teclear los datos en el ordenador, con la consiguiente pérdida de tiempo y posibilidad de errores, que se traduce en elevados costes administrativos.

Otra solución más sofisticada, pero menos frecuente, consiste en enviar dichos documentos a través del correo electrónico. El correo electrónico permite enviar mensajes entre diferentes ordenadores que estén conectados a redes de ordenadores como por ejemplo Internet o Compuserve. Sin embargo, este procedimiento no está exento de inconvenientes, debido a que el correo electrónico no está normalizado y, salvo que el cliente y el proveedor acuerden previamente componer de alguna forma sus mensajes, exige que el receptor traduzca los documentos recibidos. Además, el uso de mero correo electrónico no es un medio seguro para realizar las transmisiones comerciales ni garantiza su confidencialidad.

La solución que desde hace varios años están adoptando muchas empresas se denomina EDI o intercambio electrónico de datos. El EDI consiste en transmitir electrónicamente documentos comerciales y administrativo-contables entre aplicaciones informáticas en un formato normalizado, de forma las empresas puedan procesar dichos documentos sin intervención manual. En este trabajo describimos los sistemas de información electrónicos, las diferentes normas EDI que existen, los beneficios y los costes que para una empresa puede suponer el implantar estos sistemas y el impacto que puede tener sobre la gestión empresarial. 


\section{Transmisión de documentos administrativo-contables interem- presas}

Así como en los años ochenta y noventa el ordenador ha sido la herramienta de trabajo básica en la gestión empresarial, desde hace varios años asistimos a una verdadera revolución en la transmisión electrónica de información, de forma que se intuye que en los umbrales del siglo XXI las herramientas que utilizará el economista estarán ligadas a las telecomunicaciones. En palabras de René Ricol (1995), presidente de la orden de expertos contables de Francia, "de todos los progresos que acompañarán a la profesión contable, el EDI es ciertamente el más inminente".

El sistema tradicional en el que se basan las transmisiones de documentos entre las empresas, al estar centrado en el uso del papel, presenta dos inconvenientes. En primer lugar, la lentitud: documentos que se generan en ordenadores que procesan la información a gran velocidad, posteriormente sufren retrasos al tener que imprimirse, manipularse en las empresas de correos e introducir manualmente sus datos en los sistemas de información de clientes y proveedores. Aunque muchas empresas han sustituído el correo como medio para enviar sus documentos por el fax, lo que ha agilizado en buena medida la gestión, se mantiene otro problema: la diversidad de modelos de facturas, albaranes, pedidos, hojas de precios, etc. Esta falta de normalización es causa de muchos errores administrativos y, de nuevo, de lentitud.

El EDI consiste en incorporar un procedimiento electrónico de transmisión de información al documento administrativo-contable. La empresa puede obtener ahorro de varias formas tras implantar un sistema electrónico de intercambio de datos. En primer lugar, aparece un ahorro de tiempo, ya que la información viaja por redes de comunicación. En segundo lugar, se producen menos errores, ya que el proceso está completamente automatizado. Además puede haber un importante ahorro en dinero, dependiendo de lo que cueste enviar estos documentos. A continuación resumiremos toda la operativa necesaria para una operación comercial internacional. Ilustramos esta operativa bien conocida, para reflexionar sobre la cantidad de comunicaciones y papeleo necesario para llevar a cabo una simple operación comercial. En este apartado seguimos el manual "Presentación del EDI", editado por la Comisión de las Comunidades Europeas (1989).

Supongamos una empresa que desea adquirir un producto. Como vemos en el esquema de la figura 1, lo habitual es que envíe una petición de ofertas a sus proveedores. Los proveedores envían sus ofertas al cliente, confirman cantidades, fechas de entrega o incluso proponen alternativas, con lo que se produce un flujo de información en los dos sentidos. Finalmente, si el cliente y el proveedor llegan 
a un acuerdo, se plasma en un pedido. Normalmente el proveedor responde confirmando el pedido o realizando alguna modificación al mismo.

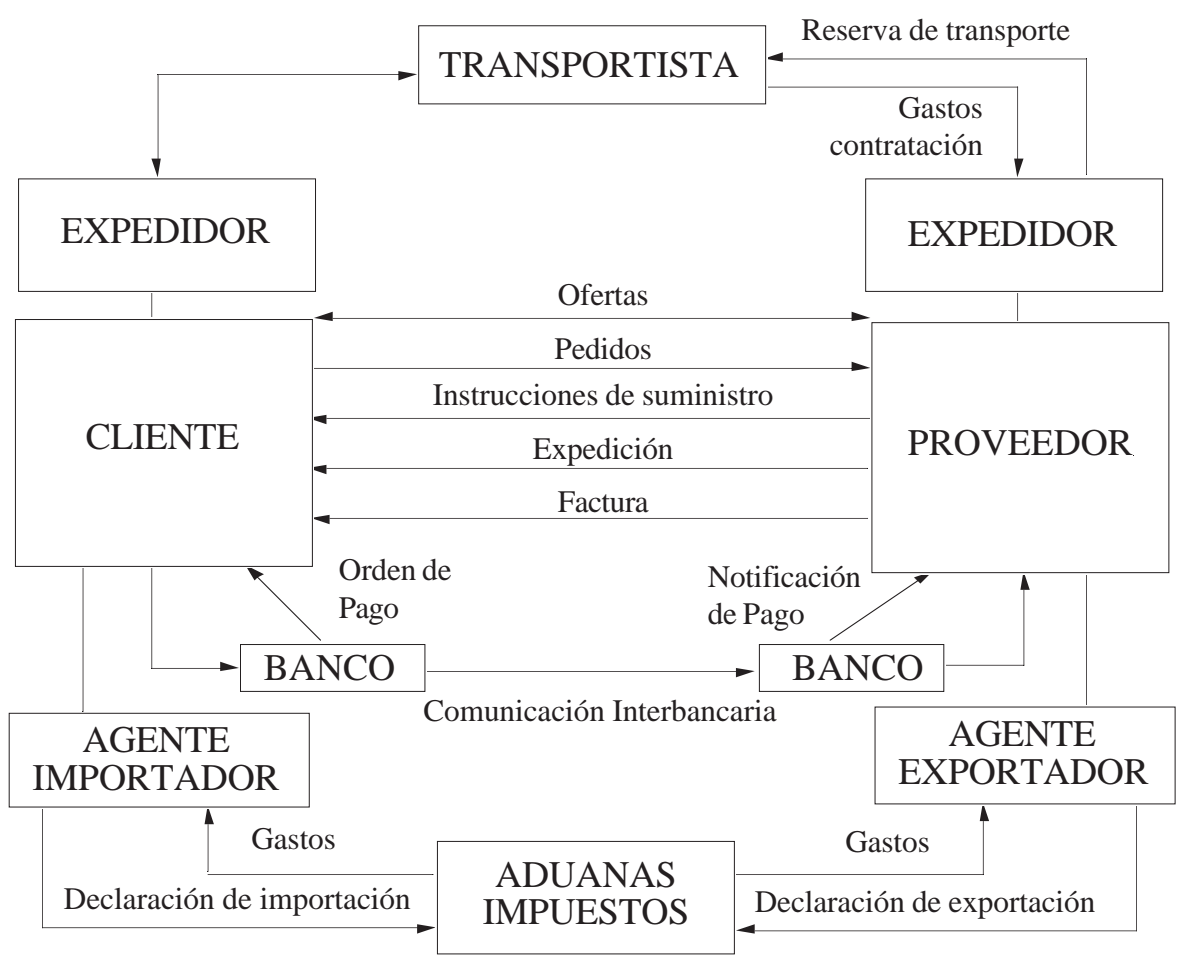

Fig. 1. Los principales intercambios de documentación entre los interlocutores.

Cuando las mercancías están listas, el proveedor envía al comprador un aviso de expedición, que informa de que el pedido está en camino o de que puede ir a recoger la mercancía. Una vez recibida la mercancía, el cliente puede notificarlo al proveedor enviando el correspondiente albarán. A continuación, el proveedor envía al cliente la factura; además puede enviar un estado de cuenta periódico en el que figuren los pagos pendientes. El cliente y el proveedor también pueden enviarse recíprocamente notas de abono y de cargo para corregir las facturas por causa de error en los precios o las cantidades, de devoluciones o deterioros en la mercancía, etc. 
Seguidamente, el cliente puede pagar al proveedor a través de una orden de pago enviada a su propio banco. También puede informarle de que el pago está en marcha. A continuación, el banco del cliente paga al banco del proveedor. Cuando éste recibe los fondos, notifica al proveedor un abono en el que se detallan las facturas que han sido pagadas.

En un momento determinado del ciclo comercial, el cliente o el proveedor, solicita una reserva de los servicios de transporte a un transportista basándose en los datos iniciales de la consignación. El transportista puede enviar al solicitante un mensaje en el que se relacionen los servicios de transporte y afines prestados y se solicite el pago de los mismos. La orden de pago se efectúa de manera análoga a la descrita anteriormente.

Cuando una transacción exija la intervención de las autoridades aduaneras, estas reciben notificación del exportador, importador y transportista sobre el movimiento de mercancías a través de la frontera. El transportista, o su agente, remitirán la "declaración del transportista". Posteriormente se comunica al exportador, importador y transportista el importe de los derechos que haya que abonar. El importador efectúa la liquidación de los mencionados derechos a la aduana a través de los procedimientos de pago explicados anteriormente. Si resulta necesario, las aduanas pueden solicitar al importador que les remita una declaración detallada en la que figuren cada uno de los artículos para permitir la inspección de la mercancía de un envío.

\section{El Intercambio electrónico de datos en la técnica contable}

Como hemos visto, cualquier operación comercial exige de un elevado intercambio de documentos entre las empresas. Por lo tanto se hace necesario automatizar estos intercambios de documentos y normalizarlos, es decir emplear un lenguaje común.

\subsection{El EDI, lengua de los negocios}

Si se ha definido la Contabilidad como "lenguaje de los negocios", podemos definir el EDI como una "lengua de los negocios". De la misma manera que en para un sólo lenguaje, el lenguaje humano, existen varias lenguas o idiomas, el contable, para comunicarse mediante el lenguaje de la Contabilidad, dispone de varias lenguas, la lengua del papel y los libros contables, la lengua del ordenador y los programas informáticos y esta nueva lengua, el EDI y las telecomunicaciones.

Para hablar la lengua del EDI, el contable debe disponer de un equipamiento y aprender unas reglas gramaticales. Los equipos necesarios para incorporar el EDI varían muchísimo: desde un simple ordenador personal en el que se instala el programa EDI a un equipo grande que gestiona las comunicaciones. También

Scire. 2 : 2 (jul.-dic. 1996) 79-98 
los programas EDI admiten muchas posibilidades, en función del nivel de integración con el resto de aplicaciones informáticas de la empresa. Hoy en día, lo más habitual es que el programa EDI se encargue de traducir los formatos del programa de contabilidad a las normas EDI y viceversa. Posteriormente, el módulo de comunicaciones del programa EDI actúa de forma semejante a cualquier programa de correo electrónico enviando los mensajes. Algunos fabricantes de programas de contabilidad están empezando a incorporar módulos que realizan funciones EDI. Esta es la situación que pensamos será más extendida a medio plazo, de la misma forma que los fabricantes de procesadores de texto $\mathrm{u}$ hojas de cálculo incorporan en sus programas módulos que permiten enviar correo electrónico.

Intercambiar datos a través de ordenadores apartados físicamente es perfectamente posible con una aplicación como el correo electrónico. La aportación que supone el EDI es el intento de normalizar todos los documentos comerciales. Ampliando el ejemplo de Ritchie (1994) podemos establecer un paralelismo entre el envío de un documento mediante EDI o utilizando una carta.

- En la carta existe un conjunto de reglas gramaticales que determinan el vocabulario aceptado y el orden en que se pueden usar las palabras y frases. En los sistemas EDI existen también unas reglas de sintaxis para la adecuada estructuración de los caracteres admitidos. Concretamente, las reglas gramaticales del EDIFACT, el estándar EDI amparado por las Naciones Unidas son una norma ISO 9735, emitida por la International Organization for Standarization.

- Tanto en la carta como en el EDI es necesario utilizar un vocabulario de términos aceptados. Si en el lenguaje humano hablamos de palabras, oraciones, etc., en el EDI hablaremos de datos, segmentos, mensajes y códigos estándares.

Los datos son la unidad más pequeña, y, siguiendo nuestro ejemplo, equivalen a las palabras. La fecha de entrega, el número de artículos o la forma de pago son datos. Para cada estándar EDI existen directorios de elementos de datos comerciales que contienen los bloques elementales utilizados en la definición de los mensajes normalizados. Por ejemplo, dentro del EDIFACT este directorio se encuentra regulado como norma ISO 7332. Una unidad más grande que el dato es el segmento, que son grupos de datos relacionados entre sí. El directorio de segmentos contiene los segmentos normalizados, por ejemplo, los correspondientes a nombre y dirección.

Un grupo de segmentos colocados correctamente de acuerdo con una reglas de sintaxis forman un mensaje EDI. Existen directorios que contienen los mensajes normalizados correspondientes a determinadas funciones comerciales. Por 


\begin{tabular}{|c|c|c|}
\hline CONITT & $\begin{array}{l}\text { Invitation to Tender } \\
\text { Message }\end{array}$ & $\begin{array}{l}\text { El cliente expone sus necesidades a } \\
\text { los proveedores y los invita a que } \\
\text { hagan sus ofertas. }\end{array}$ \\
\hline CONTEN & Tender Message & $\begin{array}{l}\text { Mensaje que contiene la oferta del } \\
\text { proveedor. }\end{array}$ \\
\hline ORDERS & $\begin{array}{l}\text { Purchase Order } \\
\text { Message }\end{array}$ & $\begin{array}{l}\text { Contiene la información sobre el } \\
\text { pedido. }\end{array}$ \\
\hline ORDRSP & $\begin{array}{l}\text { Purchase Order } \\
\text { Response Message }\end{array}$ & $\begin{array}{l}\text { Respuesta del proveedor al cliente } \\
\text { sobre el pedido. }\end{array}$ \\
\hline DESADV & $\begin{array}{l}\text { Despatch Advice } \\
\text { Message }\end{array}$ & Aviso de expedición. \\
\hline INVOIC & Invoice Message & $\begin{array}{l}\text { Mensaje de factura, en el que figuran } \\
\text { cantidades, precios, cantidades, etc. }\end{array}$ \\
\hline CURRAC & $\begin{array}{l}\text { Current Account } \\
\text { Message }\end{array}$ & $\begin{array}{l}\text { Mensaje de estado de cuenta en el } \\
\text { que figuran los pagos pendientes. }\end{array}$ \\
\hline PAYORD & $\begin{array}{l}\text { Payment Order } \\
\text { Message }\end{array}$ & $\begin{array}{l}\text { Mensaje de orden de pago enviado al } \\
\text { propio banco del proveedor. }\end{array}$ \\
\hline CREADV & Credit Advice Message & $\begin{array}{l}\text { Cuando el banco del proveedor recibe } \\
\text { los fondos, envía un mensaje de } \\
\text { notificación de abono en el que se } \\
\text { detallan las facturas que han sido } \\
\text { pagadas. }\end{array}$ \\
\hline COITON & $\begin{array}{l}\text { Container Inland } \\
\text { Transport Order Notice }\end{array}$ & $\begin{array}{l}\text { Mensaje que envía quien solicita el } \\
\text { transporte, al transportista. }\end{array}$ \\
\hline COITOS & $\begin{array}{l}\text { Container Inland } \\
\text { Transport Order } \\
\text { Response }\end{array}$ & Respuesta del transportista. \\
\hline COITOR & $\begin{array}{l}\text { Container Inland } \\
\text { Transport Order }\end{array}$ & $\begin{array}{l}\text { Solicitud de la reserva de transporte } \\
\text { en firme. }\end{array}$ \\
\hline CUSDEC & $\begin{array}{l}\text { Customs Declaration } \\
\text { Message }\end{array}$ & $\begin{array}{l}\text { Declaración aduanera del declarante } \\
\text { (exportador o importador). }\end{array}$ \\
\hline CUSCAR & $\begin{array}{l}\text { Customs Cargo Report } \\
\text { Message }\end{array}$ & $\begin{array}{l}\text { Declaración aduanera del } \\
\text { transportista. }\end{array}$ \\
\hline
\end{tabular}

Tabla 1. Algunos mensajes EDI utilizados en el estándar EDIFACT.

ejemplo, podemos encontrar en Internet en Schlieper (1995) una guía con los mensajes del EDIFACT. Están normalizados los mensajes de pedidos, facturación, despachos de aduanas, conocimientos de embarque, etc. Por ejemplo, para los pedidos, el EDIFACT utiliza el mensaje ORDERS. Siguiendo a Teodoro 
(1994, p. 27), en el ejemplo que hemos expuesto anteriormente para describir los intercambios de documentos entre las empresas, intervendrían básicamente los mensajes de la tabla 1.

En el caso concreto de la información contable, la organización Edificas Europe, (EDI in the Finance, Informational, Cost, Accounting/Auditing and Social area) es la encargada de desarrollar mensajes relacionados con la Contabilidad, y hasta la fecha ha elaborado varios mensajes: CHACCO (Plan de cuentas), ENTREC (Asientos de diario), BALANC (Balance de Comprobación), INFENT (Cuentas Anuales), CHAMAP (Estructura para agregar varias cuentas), LEDGER (Libro Mayor), DEMDOC (Petición de documento contable) y PRODOS (Información clave sobre el cliente). En España, es el IACJCE (Instituto de Auditores Censores Jurados de Cuentas) la organización que participa en Edificas Europe.

Finalmente el conjunto de códigos estándares contiene una lista de códigos internacionales aceptados de países, monedas, condiciones de entrega, condiciones de pago, modos de transporte, tipos de embalaje, etc. La figura 2 ejemplifica la sustitución de la tradicional carta de pedido por el mensaje ORDERS, que contiene la información sobre un pedido.

Siguiendo con nuestro ejemplo, otros elementos necesarios para lograr una

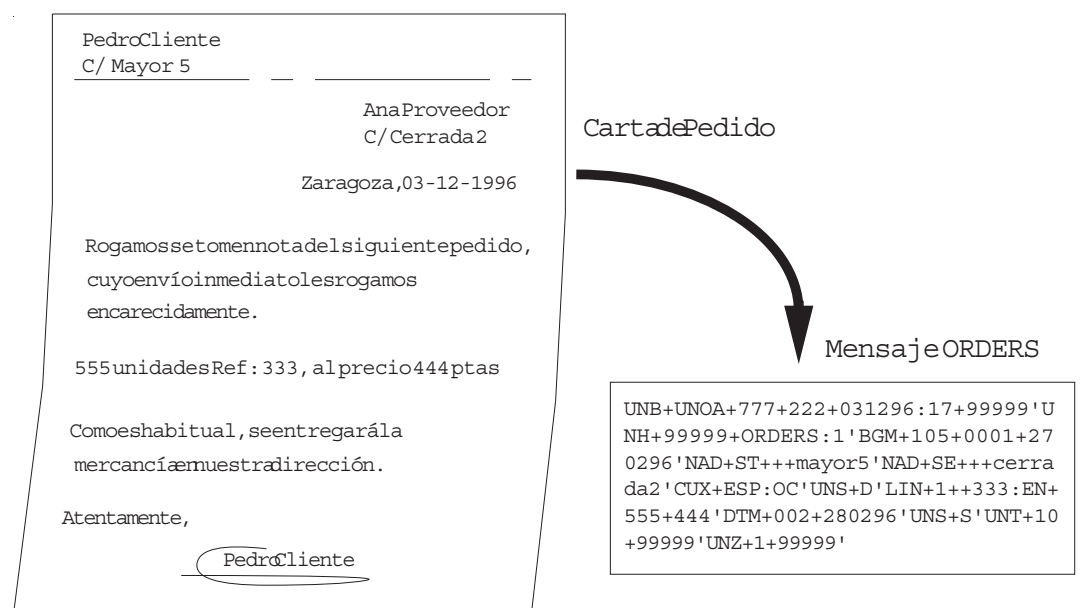

Fig. 2. De la carta de pedido al mensaje ORDERS del EDI. 
comunicación exitosa son el sobre en el que se envía la carta, el buzón y el servicio de correos. En este tipo de comunicación son necesarias unas reglas que afectan al tamaño del sobre, el sello, el lugar donde se deposita la carta, etc. En telecomunicaciones se utiliza el término protocolo, palabra que evoca los ceremoniales diplomáticos: el modelo X-25 o el X-400 son ejemplos de protocolos de red. La normalización de los protocolos de telecomunicaciones no compete a normas como el EDIFACT, aunque es indispensable para el funcionamiento del EDI. En este sentido, destacaremos que en 1992 el Comité Consultivo Internacional Telegráfico y Telefónico (CCITT) ha considerado al protocolo de correo electrónico X-400 como el estándar óptimo para los servicios EDI.

Desde el punto de vista técnico, el EDI consiste en enviar datos a través de redes y circuitos entre dos ordenadores distantes. Por lo tanto, en principio, cualquier sistema de telecomunicaciones es válido. Los servicios de red más comunes que puede utilizar una empresa para intercambiar documentos EDI son:

- Transmisión de datos por la red telefónica básica. El uso de este servicio no requiere ninguna contratación adicional a la del servicio telefónico, las partes se comunican mediante modem a través de lineas telefónicas normales con tarifas normales. Puede ser válido para un grupo pequeño de usuarios EDI, aunque la velocidad de transmisión es bastante lenta y frecuentemente surgen problemas de compatibilidad al aumentar el número de usuarios.

- Redes públicas de transmisión de datos. En España existen varias redes públicas de transmisión de datos, como Ibermic, Iberpac o RDSI. Dentro de un mismo país la utilización de estas redes no constituye normalmente ningún problema para el EDI, pero en el ámbito internacional pueden surgir dificultades, ya que no todos los países aplican las normas comunes en la misma medida. Además, hay una total ausencia de apoyo y servicio a los usuarios.

- Servicios de Valor Añadido: Los Servicios de Valor Añadido son servicios de telecomunicación que satisfacen nuevas necesidades específicas de telecomunicaciones como pueden ser en el caso de la voz, la telefonía móvil automática; en el caso de las imágenes, la videoconferencia o en el caso de los datos, el EDI. Utilizar los Servicios de Valor Añadido para el EDI presenta como ventaja el apoyo prestado al usuario. El principal inconveniente es que resulta una opción cara, por lo que previamente hay que determinar con precisión qué servicios vamos a necesitar, por ejemplo el grado de conexión internacional o la posibilidad de conexión con otras redes. Si el sistema EDI está llamado a sustituir las facturas tradicionales del tráfico mercantil por una facturación vía telemática, y ha de ser válido desde el 
punto de vista jurídico-fiscal una pieza clave es el llamado centro servidor, entidad de servicios de telecomunicación de valor añadido. Siguiendo a Jacobo (1996), el centro servidor centraliza y distribuye los mensajes entre los usuarios y es quien asegura la efectividad, transparencia o integridad de los datos transmitidos entre los usuarios.

- Redes privadas. Son utilizadas normalmente por multinacionales con enormes volúmenes de datos que necesitan garantizar su seguridad y alquilan a las administraciones nacionales de telecomunicación de uno o varios países las líneas necesarias que enlazan sus dependencias. Aparte del elevado coste de las líneas, existe el coste de los especialistas necesarios para establecer y mantener los servicios de la red y prestar ayuda a los usuarios en todos los puntos.

\subsection{Situación actual de las diferentes normas EDI}

En 1968 las empresas de transporte de Estados Unidos crearon el TDCC (Transport Data Coordinating Committee), que en 1975 dio lugar al primer conjunto de normas conocidas como EDI. A raíz de esta exitosa experiencia, el American National Standards Institute desarrolló la estandarización de los documentos mercantiles conocida con el nombre de ANSI X12. Otro hito importante en la breve historia del EDI sucede en 1986, cuando el comité de trabajo de las Naciones Unidas conocido como WP4 empezó a trabajar en la preparación de una norma de sintaxis internacionalmente aceptable para la transferencia de mensajes electrónicos. Fruto de este trabajo es la norma EDIFACT, cuyas siglas significan EDI For Administration, Commerce and Transport. La International Organization for Standarization emite los acuerdos del comité WP4 como normas ISO.

Estas normas EDIFACT fueron paulatinamente adoptadas por los estados europeos, del Pacífico, Australia, Japón, etc. En 1988 la administración de aduanas de Estados Unidos declaró su intención de respaldar la norma EDIFACT. Mientras tanto, las compañías norteamericanas seguían involucradas en el perfeccionamiento de sus propias normas X12, al margen de estos esfuerzos normalizadores a nivel mundial. Esto les empezó a originar problemas, ya que debían mantener dos sistemas: en las relaciones internas el X12 y en las importaciones y exportaciones el EDIFACT, motivo por el cual en Estados Unidos hay muchos deseos de converger sus normas ANSI X12 hacia las normas EDIFACT. En España, en 1990, la norma UNE 1145/90 define las reglas de sintaxis a nivel de aplicación, conteniendo la norma europea EN 29735 adoptada por el Comité Europeo de Normalización (CEN) en noviembre de 1989. 
A pesar de estos intentos de crear mensajes normalizados de carácter universal, esta normalización no es universal ya que coexisten diferentes sistemas EDI. Hay empresas que utilizan sus propios sistemas EDI para su uso interno o para sus relaciones con grandes clientes, que generalmente consisten en adaptaciones de EDI ya existentes. Según Ritchie (1994), normalmente estos sistemas se basan en correo electrónico y son utilizados de una forma esporádica y en grupos reducidos, aunque ha sucedido en ocasiones que a partir de ahí ha crecido el grupo y empiezan a surgir problemas de incompatibilidad. Como consecuencia, muchos sectores de la actividad económica utilizan sus propios EDI. De esta forma, nos podemos encontrar casos como el de SEAT, expuesto por Mateo (1993). SEAT utiliza un EDI con norma propia entre sus fábricas, concesionarios y el resto del consorcio VW, AUDI y SKODA. Con sus proveedores nacionales y extranjeros no alemanes utiliza EDI con norma ODETTE. Finalmente, utiliza la norma VDA con los proveedores alemanes.

\section{Oportunidades y riesgos del intercambio electrónico de datos}

Podemos agrupar los elementos positivos y negativos que supone para la empresa incorporar un sistema EDI en varios apartados, según sean factores inherentes a la gestión de la documentación de la empresa, factores logísticos, estratégicos y organizativos, legales y de seguridad (Pozo et al, 1995).

\subsection{Factores relativos a la gestión de los documentos}

El EDI permite destinar menos recursos humanos a tareas administrativas. Keegan y Portik (1995) ofrecen en su artículo su visión de la profesión contable para el siglo XXI. En su panorámica, las tecnologías de la comunicación afectarán notablemente a las actividades típicas de la gestión empresarial. En su opinión, disminuirá el personal ocupado en tareas administrativas dentro del departamento de contabilidad. Los pagos y cobros electrónicos serán tan frecuentes que los departamentos de cuentas a cobrar y pagar serán invisibles. Las conciliaciones de saldos se automatizarán debido a la popularización de programas que comprueban el saldo del ordenador de la empresa con el saldo del ordenador del banco; los problemas que surjan aparecerán en la pantalla y serán resueltos desde los propios departamentos de compras o ventas. También el EDI permite la automatización de parte de la contabilización de los asientos relacionados con aspectos comerciales.

Utilizar EDI supone un paso adelante en viejo sueño: la oficina sin papel. En los últimos años, la ofimática u oficina informatizada ha experimentado un notable desarrollo, debido no sólo al bajo precio de los ordenadores personales sino a la utilización de entornos de trabajo altamente virtualizados, basados en la metáfora del escritorio, según la cual la pantalla del ordenador es la mesa de trabajo.

Scire. $2: 2$ (jul.-dic. 1996) 79-98 
De esta forma, el usuario, sea administrativo o directivo dispone en su ordenador de un verdadero escritorio, con agenda, procesador de textos, papelera, calculadora, correo electrónico, etc. El EDI es un elemento más que se suma al sistema de información de la oficina.

El EDI permite aumentar simultáneamente la rapidez en la gestión y reducir el número de errores. Con procedimientos convencionales de elaboración de la información, satisfacer el requisito de oportunidad de la información contable, produce a menudo una disminución de la fiabilidad, como señala Gabás (1991, p. 69). Una solución para que la información sea oportuna, sin perder fiabilidad, pasa por la incorporación de las nuevas tecnologías de las telecomunicaciones a la empresa, como el EDI, que permite reducir el número de errores en las facturas. Según Manuel Pereira, presidente de Simpro España, el 50\% de las facturas en nuestro país contienen al menos un error, (Expansión ,1993). Esto es posible porque los datos recibidos directamente de ficheros y aplicaciones informáticas contienen menos errores que los que se introducen a mano. Por otro lado, los datos transmitidos por el ordenador se procesan más rápidamente que los enviados por correo e introducidos manualmente.

El EDI también tiene unos costes de implantación, además del ordenador y modem, entre estos costes destaca el del software EDI y del contrato de mantenimiento. Otro coste propio del EDI es el alta en el centro de compensación. No menos importantes son los costes de formación del personal, y las adaptaciones en el software de la empresa o software de integración. Frecuentemente hay que rediseñar las aplicaciones informáticas utilizadas en la empresa para enlazarlas con el sistema EDI; aunque esto supone un coste también es una oportunidad para mejorarlas.

El coste de explotación del EDI normalmente es menor que el de los sistemas tradicionales, como el correo y el fax. En general, las transmisiones son muy cortas por lo que las tarifas de uso de líneas telefónicas son más moderadas. En ocasiones se paga un canon que es independiente del uso que se hace de las mismas. Entre los costes de explotación también hay que incluir las tarifas mensuales del centro de compensación.

\subsection{Factores logísticos, estratégicos y organizativos}

El EDI favorece el just in time. Este sistema de planificación exige tal sincronización entre cliente y proveedor para que los pedidos estén siempre a tiempo que necesita el soporte de un sistema de información interempresarial como el EDI, que permite que varias organizaciones funcionen como si se tratara de una sola. Gracias a la rapidez con que se gestionan los pedidos mediante EDI, las empresas pueden reducir al mínimo sus existencias en almacén, lo que origina un importantísimo ahorro de costes de mantenimiento de inventarios. 
El EDI permite un mejor control del movimiento de los activos de la empresa. Actualmente el grado de automatización de los almacenes es muy elevado, de forma que se facilita el control de las existencias. Pero también los activos fijos son susceptibles de controlarse automáticamente. Keegan y Portik (1995) en su artículo futurista describen cómo los activos fijos de la empresa (maquinaria, automóviles, etc.) disponen de un pequeño chip con memoria y capacidad para enviar mensajes al ordenador central sobre su situación y estado actual.

Con el EDI se logran ventajas aduaneras y en el transporte. Al presentar de antemano los documentos se facilita la tramitación administrativa de los bienes que se transportan y reducen las paradas en los puestos aduaneros, fiscales y sanitarios. Incluso se ha creado y normalizado el Documento Único Aduanero como parte del EDI.

El EDI puede contribuir a mejorar la imagen y el servicio ante los clientes. Gracias a la disminución de errores en el proceso, los pedidos de los clientes son atendidos con mayor eficiencia. Además, el que haya unas relaciones más fluidas entre clientes y proveedores permite nuevas oportunidades. Andreu (1991, p. 3) describen el caso de la cadena de supermercados de juguetes Child World, que consiguió que uno de sus mayores proveedores, Fisher Price, le concediera descuentos y tratos preferenciales por comunicarles la evolución de las ventas de sus productos día a día, obtenida directamente de los terminales punto de venta en el supermercado. Fisher Price usa dicha información para afinar sus campañas publicitarias. Como vemos, en este caso el cliente es a su vez proveedor de información, y esa información tiene un precio, que se materializa en el descuento obtenido.

El EDI puede considerarse una ventaja estratégica para las empresas que disponen de este sistema, y una barrera de entrada a aquellas organizaciones que no funcionen con EDI. En sectores económicos en los que existe una gran empresa y muchos proveedores, la empresa grande puede imponer a éstos que sean capaces de utilizar el EDI. Sin embargo, hay que ser prudentes porque también el EDI puede producir monotonía y pérdida de identidad en las relaciones comerciales. Esta pérdida de identidad puede llegar a extremos verdaderamente peligrosos, como la toma de decisiones automática. Hoy en día, cualquier persona da instrucciones a su banco para que compre o venda valores con unas condiciones. Nada impide que se diseñen programas de Inteligencia Artificial con plena capacidad para tomar estas u otras decisiones de compra-venta. Es viable que sea un agente informático quien busque las ofertas más atrayentes, por ejemplo en redes como Internet y posteriormente realice el pedido utilizando el EDI. Estos programas, desarrollados en los servicios de inteligencia, son entrenados para rastrear de forma inteligente entre las bases de datos de todo el mundo.

Scire. 2 : 2 (jul.-dic. 1996) 79-98 
El EDI facilita las relaciones comerciales con otros países. El EDI es una lengua común que permite salvar las barreras del idioma, gracias a la normalización de los mensajes. Sin embargo en otras ocasiones actúa como freno, por ejemplo, algunas empresas americanas que deseaban entrar en el mercado europeo y utilizaban las normas americanas X12 encontraron muchos problemas para adaptarse al EDIFACT.

Si bien es posible que una empresa utilice EDI simplemente como medio de comunicarse con otras, en general el EDI se integra con las aplicaciones del usuario. Esta forma de aplicar el EDI exige que los sistemas de información internos de cada empresa se adapten a la información que tienen que enviar y recibir. En definitiva, se incrementa la disciplina interna en la formalización de los datos. Dando un paso adelante, el EDI permite realizar una reingeniería de los procedimientos (Teodoro, 1994, p. 62). En resumen, al implantar sistemas EDI se consiguen mejoras en la disponibilidad de la información para la toma de decisiones, ya que aumenta la integración de información e informática.

El EDI produce un importante cambio cultural en la empresa. Para del Pozo et al. (1995), el EDI supone un 75\% de esfuerzo a nivel de organización y un $25 \%$ a nivel técnico. También desde el punto de vista de la organización pueden surgir problemas al incorporar un sistema EDI. Inicialmente se pueden producir desajustes organizativos, ya que el EDI representa una filosofía de empresa que requiere que se recicle buena parte del personal, con todo lo que supone de traumático el cambio.

\subsection{Factores relativos a la seguridad}

Las empresas que utilizan sistemas de información llegan a ser, casi inevitablemente, dependientes de ellos, lo cual es un arma de doble filo, pues se corre el riesgo de que un fallo provoque el caos. Toda actividad de la empresa que, como el EDI, se apoye en equipos informáticos, debe reducir al máximo los riesgos derivados de la falta de seguridad y del uso inadecuado o fraudulento de la información. La seguridad de los mensajes EDI es un asunto de vital importancia y tiene un doble objetivo: garantizar que los mensajes llegan a su destino y mantener la confidencialidad de los mismos. Para garantizar la autenticidad del envío existen las Autoridades de Certificación que actúan como terceros para que las empresas estén seguras de la autenticidad de los mensajes recibidos. Los mensajes se pueden precintar para asegurar que su contenido no se ha modificado, ni accidentalmente ni deliberadamente.

Otro aspecto que tiene que ver con la seguridad es la confidencialidad de la información: por todos son conocidos casos de empleados que malversan fondos de la propia empresa aprovechando debilidades en la seguridad del sistema informático. Hay que garantizar que los datos sean modificados o consultados sólo por 
aquellos usuarios autorizados, para ello se trazan mapas o perfiles de usuario, es decir, se trata de regular grupos de acciones autorizadas a cada colectivo de usuarios. También se definen palabras clave para acceder al sistema. Otra norma básica es evitar que una sola persona tenga el control de todas las funciones EDI, al fin y al cabo también en un sistema manual se suelen tomar garantías como que sean dos las personas que firmen los cheques.

Para verificar si se están aplicando las medidas de control más apropiadas para la salvaguarda e integridad de la información y de los sistemas en prevención de riesgos de fraude, pérdida, manipulación, fallos de servicio, etc., el papel de la auditoría informática, como revisión profunda y detallada de todos los elementos de que dispone una empresa en el área de sistemas de información es fundamental.

\subsection{Implicaciones legales y fiscales del EDI}

La legislación de todos los países en materia de facturación telemática está adaptándose para evitar el vacío legal existente. En 1991, la Asociación Española de Codificación Comercial (AECOC) planteó a la Agencia Estatal de la Administración Tributaria, la conveniencia de autorizar un sistema de teletransmisión de facturas. Esta petición tuvo una favorable acogida, que se materializó en el Real Decreto 1.624/1992, que modifica al 2.402/1985 que regulaba el deber de expedir y entregar facturas. El nuevo decreto indica que las facturas enviadas por vía telemática tienen la misma validez que las originales, siendo únicamente necesaria que las partes consientan en utilizar este tipo de transmisión de los documentos, amén de una autorización de la administración tributaria. Sus nueve apartados son:

1) Las facturas emitidas por vía telemática tendrán la misma validez que las facturas originales, debiendo ser la información de la factura emitida y recibida, idéntica.

2) La Administración Tributaria podrá exigir en cualquier momento, al empresario o profesional emisor o receptor, su transformación en lenguaje legible -por métodos ordinarios-, así como su emisión en soporte de papel.

3) Los empresarios o profesionales o sus agrupaciones, que deseen utilizar el sistema de facturación telemática deberán solicitarlo a la Agencia Estatal de Administración Tributaria, indicando los elementos que permiten comprobar que el sistema de transmisión a distancia propuesto cumple las condiciones exigidas en este artículo.

4) La Agencia Estatal resolverá sobre la solicitud en el plazo de los seis meses siguientes a su recepción, sin perjuicio del requerimiento de 
cuantos datos o nuevas informaciones resulten necesarios para la resolución del expediente, en cuyo caso se interrumpirá dicho plazo.

5) Las modificaciones del sistema ya autorizado anteriormente deberán comunicarse previamente a la Agencia Estatal, entendiéndose aceptadas si no se deniegan en el plazo de los seis meses siguientes.

6) Durante la tramitación de la solicitud inicial o de la modificación del sistema, el Departamento de Inspección Financiera y Tributaria de la Agencia Estatal podrá realizar controles en el establecimiento del emisor, del receptor o del prestador del servicio de teletransmisión. Una vez autorizado el sistema, los interesados presentarán a la Administración Tributaria una solicitud de autorización de su uso con una anticipación mínima de treinta días a su puesta en servicio.

7) Las facturas deben conservarse con su contenido original y en el orden cronológico de su emisión por el empresario o profesional emisor y de su recepción por el empresario o profesional receptor en los plazos y condiciones fijados por este Real Decreto. Sólo deberán conservarse en soporte papel, durante los plazos señalados, una lista semestral de los mensajes emitidos y recibidos y de correcciones o anomalías eventuales.

8) La Administración Tributaria podrá comprobar en cualquier momento en los locales de los empresarios y profesionales autorizados así como de los prestadores del servicio de teletransmisión que el sistema cumple las condiciones exigidas en la autorización, mediante las operaciones técnicas necesarias para constatar su fiabilidad.

9) El sistema no será de aplicación hasta que el Ministerio de Economía y Hacienda dicte las normas de aplicación. Normas que se contienen en el proyecto en elaboración en este momento.

El Real Decreto 80/1996 de 26 de enero ha completado la regulación de los SIFMT (Sistema de Intercambio de Facturación por Medios Telemáticos). El esquema organizativo de un SIFMT incluye un promotor, (agrupación de empresas que solicitan la posibilidad de facturación telemática) el centro servidor (entidad de servicios de valor añadido que centraliza y distribuye los mensajes de facturación telemática), el prestador de servicios informáticos (empresario que desarrolla o comercializa los programas y equipos informáticos) y naturalmente los usuarios del sistema, que son todos los contribuyentes que quieran hacer uso del mismo. Danos y Srivam (1995) comentan para el caso de los Estados Unidos las nuevas obligaciones y cautelas que el utilizar EDI supone en el almacenamiento electrónico de los documentos. Las obligaciones de los usuarios, según Jacobo (1996), están en relación con la conservación de los soportes y afectan a 
las tareas típicas del departamento de contabilidad, por lo que las reproducimos a continuación:

a) Conservar en fichero magnético u óptico los ficheros transmitidos y recibidos.

b) Que el estado de los soportes magnéticos u ópticos permita su visualización en pantalla, o su impresión en soporte, papel o copia, en soporte magnético.

c) Conservar un listado resumen en soporte magnético u óptico y en papel, por cada transmisión efectuada o recibida. Opcionalmente podrá integrarse en un listado resumen diario todas las transmisiones efectuadas en el mismo día, conservando el orden de transmisión, con carácter secuencial.

d) Facilitar a la Inspección el acceso a sus instalaciones, a la realización de pruebas necesarias y obtención de copias de los datos en papel o soporte magnético u óptico de todos o parte de los ficheros originales, incluidas las tablas de los códigos estables, ordenar cualquier proceso informático sobre los mismos y cuantos documentos sean necesarios al objeto de facilitar el control del SIFMT y la propia situación fiscal del contribuyente.

e) A petición expresa de cualquier cliente o parte interesada, imprimir las facturas que le sean solicitas en soporte de papel.

Finalmente, las relaciones telemáticas entre las empresas y la Administración Pública y entre los propios Organismos Públicos se ha regulado en los mismos términos en la Ley 30/1992 de Régimen Jurídico y Procedimiento Administrativo de las Administraciones Públicas.

\section{Reflexión final. Impacto del Intercambio electrónico de datos}

La utilización intensiva de herramientas ligadas al sector de las telecomunicaciones introducirá cambios importantes en muchas de las actividades típicas del contable. De entre todas las novedades que ligadas a esta tecnología repercutirán en la profesión contable, el EDI es la más inminente. La captura de datos se verá facilitada con los mensajes EDI; pensemos en mensajes como ORDERS (pedido), DESADV (aviso de expedición), INVOIC (factura), INVRPT (estado de las existencias) y otros. Cuando el EDI está perfectamente integrado con el resto de aplicaciones informáticas, la mayoría de los asientos se realizan de forma automática. Actividades como la facturación o el control de las existencias se automatizan con el EDI. Finalmente, el proceso de transmisión de la información contable también se agiliza, contribuyendo a satisfacer el requisito de oportunidad de la información contable. 
Por estas razones, no es de extrañar que los profesionales de la contabilidad manifiesten temor ante una automatización de sus tareas, que reduzca los puestos de trabajo en el sector. Sobre este particular, Stoven (1995) opina que el EDI no supone una amenaza para la profesión contable. Al aumentar la fiabilidad y oportunidad de la información contable, mejorará su calidad y habrá una mayor demanda de información contable para facilitar la toma de decisiones. Alvarez (1995) explica que la organización del departamento de contabilidad de cualquier empresa pasa necesariamente por la captura y organización de los "papeles". Estamos de acuerdo con esta afirmación pero nos atrevemos a sustituir la palabra "papeles" por el término más general de "documento", independientemente de si el soporte físico es papel, un fichero informático o una sucesión de impulsos eléctricos. En nuestra opinión, al menos en la pequeña empresa, el contable que disponga de una adecuada formación puede asumir estas nuevas funciones ya que ha sido el tradicional gestor de la información empresarial.

A medio plazo, el departamento financiero de las empresas puede verse seriamente afectado por el outsourcing, es decir, que sean los proveedores de la empresa quienes realicen actividades que tradicionalmente realiza el departamento contable-financiero. De hecho, hoy en día un número muy importante de pequeñas empresas confían su contabilidad a despachos profesionales. Esto provoca que haya un flujo de información no sólo entre la empresa con sus clientes, proveedores, la administración, etc. sino también con su contable. De acuerdo con Chiaramonti (1995), Vicepresidente de EDIFACT-ONU, estos despachos profesionales pueden motivar que sus clientes, generalmente pequeñas empresas, utilicen el EDI. A menudo estos despachos son para sus clientes verdaderos consejeros no sólo en los temas de contabilidad o fiscalidad, sino en las nuevas tecnologías de la información. Por esta razón, Chiaramonti (1995) desea que "los expertos contables se encuentren en primera línea para el desarrollo del EDI". Para Teodoro (1994, p. 84), la proliferación de EDI plantea en algunos sectores la aparición de ciertos intermediarios dedicados a tareas administrativas o de control de existencias. Según Keegan y Portik (1995), una de las principales tareas del departamento financiero será precisamente identificar qué compañías son capaces de realizar mejor los servicios.

Si los contables deberán reciclarse para asumir las nuevas actividades que traerá el EDI, también los auditores verán modificadas muchas de sus formas de trabajar. Para Teodoro (1994, p. 47), es previsible que la proliferación de sistemas EDI lleve a la utilización cada vez más acentuada de técnicas de auditoría asistida por ordenador (CAA). Los auditores tendrán acceso a archivos electrónicos de documentos, que pueden almacenarse en discos WORM (Write Once Read Many times). Este soporte es ideal para posteriormente realizar auditorías o inspecciones ya que que permite grabar los datos en ellos una sola vez y leerlos 
cuantas veces se desee. Ante la imposibilidad de comprobar manualmente si coinciden todos los documentos aportados con los asientos contabilizados, el auditor actualmente utiliza técnicas estadísticas; con el uso creciente de los soportes informáticos se podrá evitar el muestreo, verificando automáticamente la validez de los registros.

Otra característica de este fin de siglo es el avance imparable de la normalización en todos los aspectos de la economía. Estos intentos de normalización involucran también a la circulación de la información contable a través de las redes de comunicación. El EDI se suma a los factores que impulsan la armonización en Contabilidad, ya que los usuarios de EDI van a demandar dicha normalización de las prácticas contables.

También la Contabilidad de Gestión puede beneficiarse de esta herramienta ya que posibilita conectar los sistemas de información de dos empresas y favorece las nuevas filosofías de gestión, basadas en el just in time, target costing, etc. Hemos iniciado este artículo comentando que suele suceder que dos empresas que mantienen una intensa relación comercial cliente-proveedor y que disponen de sendos sistemas informativos contables avanzados encontraban cuellos de botella en sus comunicaciones. Con el EDI, tal y como destaca Chiaramonti (1995) puede darse la paradoja de que haya una mayor sintonía entre un subsistema de información de la empresa, como por ejemplo el de compras, con el subsistema de información del proveedor que con otros subsistemas de la propia empresa.

\section{Notas}

(1) En la siguiente dirección de Internet, correspondiente a la empresa Premenos, podemos encontrar los segmentos de dicho mensaje: URL : http://www.premenos.com/unedifact/Messages/orders.html

\section{Referencias}

Andreu, R.; Ricart. J. y Valor, J. (1991): Estrategia y Sistemas de Información. McGraw Hill, 1991.

Chiaramonti, C. (1995). La comptabilité: secteur organisé pour favoriser l'essor d'un EDI intersectoriel. // Revué Francaise de Comptabilité (Septembre 1995) 21-23.

Comisión de las Comunidades Europeas (1989). Presentación del EDI, Luxemburgo : Oficina de Publicaciones Oficiales de las Comunidades Europeas, 1989.

Danos, J.; Srivam, R.S. (1995). The IRS Regulatory Implications of Electronic Record Keeping. // The CPA Journal. (november 1995) 46-50.

Expansión (1993). Intercambio electrónico de datos: Adiós al papel. (diciembre 1993) 48-53.

Scire. 2 : 2 (jul.-dic. 1996) 79-98 
Gabás, F. (1991). El Marco Conceptual de la Contabilidad Financiera.Madrid: AECA, 1991. ( Monografía n ${ }^{\circ} 17$ de la Asociación Española de Contabilidad y Administración de Empresas)

Heath, N. (1991). Taking Account of EDI. // Management Accounting. (February 1991) 14-15.

Jacobo, J.M. (1996). Implicaciones fiscales de la factura telemática. // Partida Doble. 63 (enero 1996) 83-88.

Keegan, D.P.; Portik, S.W. (1995). Accounting will Survive the Coming Century, Won't it? // Management Accounting (December 1995 24-29

Mateo, J. (1993). El EDI en SEAT // Chip (mayo 1993) 56.

Pozo, R.; García, M.; Martínez, F.J.; Luna, P. (1995). Intercambio Electrónico de Documentos (EDI): Del avance técnico al avance contable y organizativo. // Partida Doble. 61 (noviembre 1995) 28-37.

Ricol, R. (1995). L'avenir de la profession comptable en environnement dématérialise. // Revué Francaise de Comptabilité. (Septembre 1995) 20.

Ritchie, S.K. (1994). A 'Road Map' to EDI. Curtin University of Technology. URL : <http://www.ediwi.ca:6900/Resource_Center/Agora/Roadmap/content.html>

Schlieper, H. (1995). Henry's Yellow UN/EDIFACT Book, Introduction to UN/EDIFACT Messages. URL : <http://www.premenos.com/klaus/henry/>

Stoven, B. (1995). Stratégie des comptables à l'égard d'Edifact. // Revué Francaise de Comptabilité. (Septembre 1995) 20.

Teodoro, J. (1994). Intercambio electrónico de datos (EDI). Madrid : Ministerio de Obras Públicas, Transportes y Medio Ambiente, 1994. 